\title{
Innovative Avionics Miniaturization by Chip-level Integration Using Vertical Assembly Technique
}

\author{
By Seisuke FukUdA, ${ }^{1)}$ Makoto MitA, ${ }^{1)}$ Kazuyuki Hirose,${ }^{1)}$ Masahiro KAto, ${ }^{2)}$ Takeshi MiYABARA, ${ }^{2)}$ \\ Kei SugIE, ${ }^{2)}$ Shota HARADA, ${ }^{2)}$ and Shuhei IRIYAMA ${ }^{2)}$ \\ ${ }^{1)}$ Institute of Space and Astronautical Science, JAXA, Sagamihara, Japan \\ ${ }^{2)}$ Mitsubishi Heavy Industries, Ltd., Komaki, Japan
}

(Received June 28th, 2019)

\begin{abstract}
In space exploration missions whose resources are severely restricted, miniaturization of onboard avionics by functional integration is crucial. While it seems reasonable to employ the board-level integration in the current technology level of the space community, the ultimate goal is considered to be the chip-level integration. In this paper, an innovative method for vertical assembly of various bare chips is proposed. Silicon substrates, on which bare chips are implemented, are piled up by room-temperature bonding. The room-temperature bonding is based on MEMS (Micro Electro Mechanical Systems) technology. Compared to the vertical assembly preparing the wafer-level TSV (Through Silicon Via), the method is appropriate for space-use of low-volume production because it is not necessary to develop the dedicated chips. Existing bare chips can be vertically integrated into the chip-level integrated avionics. Results of validation tests for the vertically assembled modules such as functional, vibration, and thermal tests, are also shown.
\end{abstract}

Key Words: Vertical Assembly, Chip-level Integration, Room-temperature Bonding, Miniaturization, Avionics

\section{Introduction}

In space exploration missions whose resources are severely restricted, miniaturization of onboard avionics is crucial. A variety of avionics are prepared for each individual function in common spacecraft architecture. The promising approach to miniaturize the avionics is considered to be integration. For example, there are some trials of the board-level integration where the modularized circuit boards are stacked. ${ }^{1)}$ For the further miniaturization the next technical target is supposed to be the chip-level integration. In this paper, an innovative method for vertical assembly of various bare chips is proposed. Silicon substrates, on which the bare chips are implemented, are piled up by room-temperature bonding. The room-temperature bonding is based on MEMS (Micro Electro Mechanical Systems) technology. Compared to the vertical assembly preparing the wafer-level TSV (Through Silicon $\mathrm{Via}$ ), the method is appropriate for space-use of low-volume production because it is not necessary to prepare the dedicated chips. Existing bare chips can be vertically integrated into the chip-level integrated avionics. Thus the proposed scheme seems meaningful from the viewpoint that the advanced consumer technique such as the room-temperature bonding is successfully applied with the manner of overcoming the restrictions concerning the space use. What are tried to do in this paper are summarized as:

- To establish a flexible scheme for stacking vertically heterogeneous bare chips such as MPU and SRAM by the room-temperature bonding between Silicon substrates without the wafer-level TSV,

- To manufacture several layered modules (up to four-layered modules) using the proposed scheme,
- To verify the proposed scheme against space-used circumstances by carrying out vibration and thermal tests.

In the following section, the concepts of the integration with the board-level and the chip-level methods for the avionics miniaturization are introduced again. Then the vertical assembly procedure based on the room-temperature bonding is explained in a step-by-step manner in Section 3. Results of validation tests, which are functional, vibration, and thermal tests, are described in Section 4. Finally, Section 5 gives conclusions.

\section{Avionics Miniaturization by Chip-Level Integration}

Integrating multiple functions of spacecraft avionics was tried in on-orbit satellites such as the ICU (Integrated Controller Unit) of REIMEI (INDEX), ${ }^{2,3)}$ and is employed for some spacecrafts under development such as the SMU (Spacecraft Management Unit) and IPCU (Integrated Power Control Unit) of the small lunar lander SLIM. ${ }^{4,5)}$ The integrated avionics have great advantages in the resource budgets of mass and electric power. On the other hand, when reusability and testability of the avionics is considered, some concepts of modularization become important. In the SPRINT bus for the small scientific satellites of ISAS/JAXA, avionics are standardized with the modular manner. ${ }^{6,7)}$ For example, the identical standardized computers ${ }^{8)}$ are used as not only the central data handling unit (SMU) but also the attitude and orbit control unit (AOCU) and the mission data processor (MDP) in the satellite architecture of HISAKI (SPRINT-A). Also protocols of the data interface between these avionics are standardized as SpaceWire ${ }^{9)}$ and RMAP (Remote Memory Access Protocol). ${ }^{10)}$ As indicated in the previous paper, ${ }^{6}$ a 
(1) Bump formation and coining

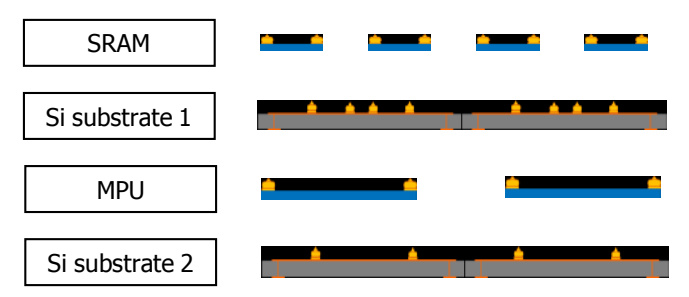

(2) Flip chip bonding and underfilling

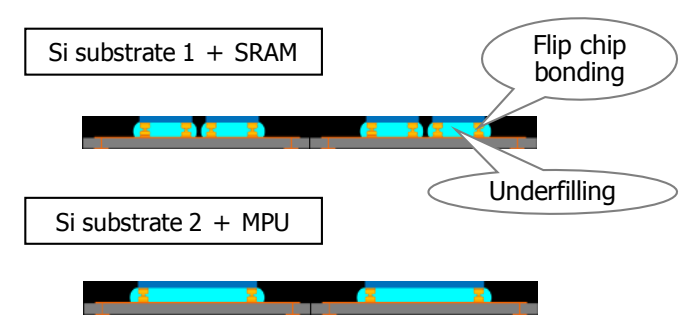

(3) Room-temperature bonding

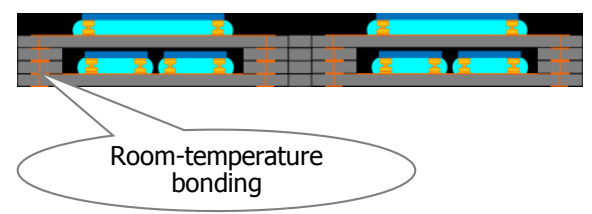

(4) Dicing



Fig. 1. Vertical assembly procedure based on the room-temperature bonding.

trade-off between the integration and modularization should be carefully considered at every development opportunity according to the technology readiness and the resource budgets including the cost.

In exploration missions with severe mass and power budgets, the integrated avionics are supposed to be essential. In the current technology level of the space community, it seems reasonable to employ the board-level integration; modularized circuit boards, with various functions such as data handling and recording, and attitude and orbit control, are stacked. The technical issues of the board-level integration are not simply stacking of the circuit boards but downsizing interface modules into a kind of palm-size unit, ${ }^{11)}$ or increasing conversion efficiency of a power supply unit.

The ultimate goal beyond the board-level is considered to be the chip-level integration. While ASIC (Application Specific Integrated Circuit) and SoC (System on Chip) can be regarded as the chip-level integration, a scheme for stacking of bare chips is investigated in this paper. The key technique is vertical assembly which piles up some bare chips vertically. Especially in this paper, the method of the vertical assembly by room-temperature bonding ${ }^{12,13)}$ is featured. Since the method has potential to integrate not only processors, memories, FPGAs (Field Programmable Gate Arrays) but also sensors like MEMS gyros and CMOS (Complementary Metal Oxide Semiconductor) image sensors, it would be able to realize the innovatively integrated miniature avionics.

\section{Vertical Assembly by Room-Temperature Bonding}

Since there are various requirements in space missions, it is not realistic to develop the dedicated bare chips for the vertical assembly. Furthermore, the development of space-qualified chips needs much higher cost per user than consumer use, so that the merit of mass production could not be applicable. Therefore, it is supposed that the common approach of the vertical assembly, which prepares the wafer-level TSV (Through-Silicon Via), is not applicable in the space avionics. On the contrary, the solution in this paper is based on the idea that the Silicon substrates with wiring patterns are stacked vertically. As the patterns of the substrates can be designed flexibly, the vertical assembly can be achieved using the existing heterogeneous bare chips without the wafer-level TSV.

In our scheme, the Silicon substrates are stacked by the room-temperature bonding. The surface of the substrates is activated by irradiation of an ion beam in vacuum because the surface oxides are ejected. Through the activated surfaces, the substrates are bonded each other by no heating process. The room-temperature process with no heating makes the substrates free from the residual heat-strain. So it is expected that the proposed scheme is robust against environmental diversities such as thermal cycles.

Figure 1 depicts an example of the vertical assembly procedure based on the room-temperature bonding. In this example, one MPU (Micro Processor Unit) and two SRAM (Static Random Access Memory) chips are assembled using four layers of the Silicon substrates. First, as (1) of Fig. 1, the bumps are formed on the substrates and chips. The bumps are made of $\mathrm{Au}$. The so-called coining process is carried out in order to flatten the top of the Au bumps. Figure 2 shows an example of a SEM (Scanning Electron Microscope) image of the Au bump after the coining.

Next, as (2) of Fig. 1, the substrates and bare chips of the MPU and SRAMs are bonded by the Au-Au flip chip assembly. After the flip chip bonding, the spaces between the 


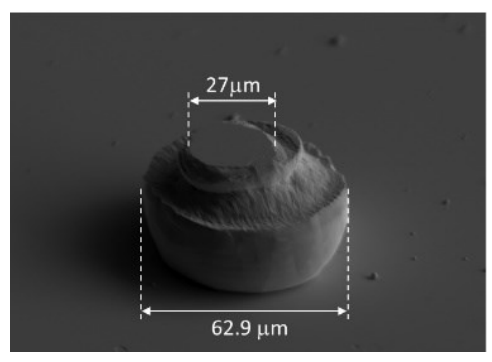

Fig. 2. Example of a SEM (Scanning Electron Microscope) image of the Au bump after the coining.

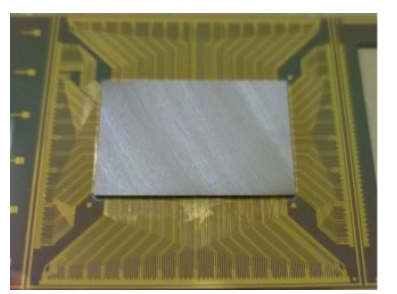

(a)

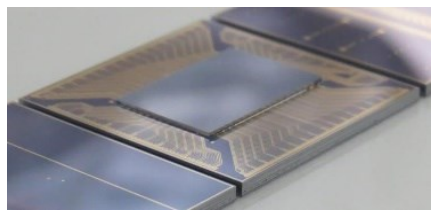

(c)

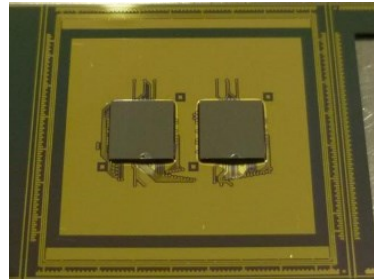

(b)



(d)
Fig. 3. Snapshots of the vertical assembly procedure: (a) substrates where the MPU is mounted, (b) substrate where the SRAMs are mounted, (c) result of the dicing, and (d) result of the wire bonding.

chip and the substrate are underfilled by the liquid curable resin. Figure 3 (a) and (b) show the substrates where the MPU and SRAMs are mounted.

After that, as (3) of Fig. 1, the substrate with the MPU, the substrate with the SRAMs, and two hollow substrates are assembled vertically. Since in each substrate through holes are manufactured by deep reactive-ion etching, the substrates are stacked layer by layer using the room-temperature bonding.

Finally, as (4) of Fig. 1, the vertically assembled substrates are cut into the individual modules by the dicing. Figure 3 (c) is a picture of the dicing result. In the case of the four-layered modules like Fig. 1, the laser-based stealth dicing can be employed. For the modules with seven and more layers, however, the stealth dicing becomes difficult to be applied because the laser power decreases both by the reflection between the air and Silicon, and by the absorption inside the Silicon substrates. Hence in such cases, an alternative dicing method using the diamond blade needs to be used. When the blade dicing is put into practice, the closest attention to the chipping (Fig. 4) should be paid not to break the modules.

After the vertical assembly, the module is mounted on an interposer as illustrated in Fig. 5. Then wire bonding is carried out from the top of the module. Figure 3 (d) is a picture after the wire bonding. Here the thickness of the Au plating for the wire bonding is an important design parameter. In the end, the module is filled by the mold, and is covered by a package.

\section{Results of Validation Tests}

According to the assembly procedure shown in Section 3, three kinds of the modules were manufactured:

- Type A: one-layered module on which the MPU is mounted (reference)

- Type B: two-layered module on which the MPU is mounted

- Type C: four-layered module on which the MPU and SRAMs are mounted (as Fig. 5)

As the bare chips mounted on the modules, the MPU and SRAM based on SOI (Silicon On Insulator) technology were selected: The SOI-SRAM ${ }^{14)}$ and SOI-SOC $2^{15)}$ by Mitsubishi Heavy Industries. The SOI-SOC2 was employed for the mission avionics of HITOMI (ASTRO-H) and ARASE $(E R G)^{16)}$ satellites, and were successfully operated on the orbits, because the SOI has superior characteristics of radiation-hardness.

In the following, the results of validation tests for these vertically assembled modules such as functional tests, vibration tests, and thermal tests.

\subsection{Functional tests}

The electrical characteristics and functional validation of the MPU at the unit level were tested using the IC tester (Advantest, T6577). Figure 6 shows a performance board to

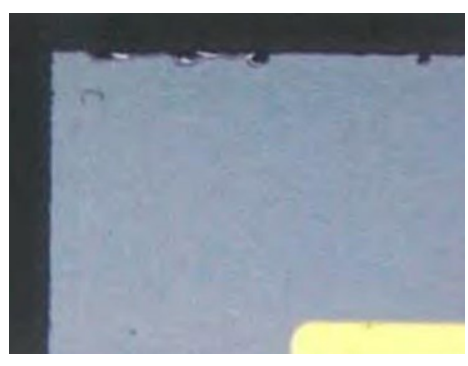

Fig. 4. Chipping due to the blade dicing.

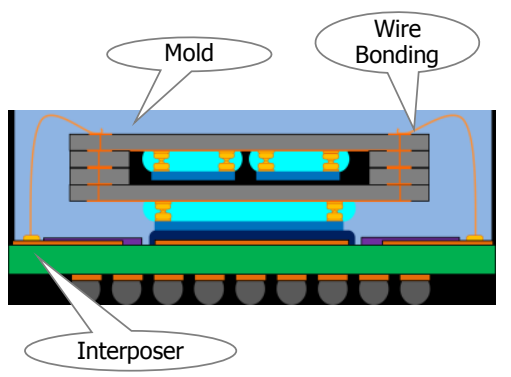

Fig. 5. Illustration of the vertically assembled module mounted on an interposer. 
interface between the IC tester and the tested modules.

For the functional validation at the module level, an evaluation board were newly developed. On the evaluation board, SRAM and SDRAM are implemented outside the module, so that the function of each interface could be verified. Also on the board there is an FPGA including a SpaceWire router. Recently a brand-new SOI-SOC, so-called SOI-SOC $3,{ }^{17,18)}$ has been developed. The SOI-SOC3 contains not only the SpaceWire router but also the SpaceWire Engine which is in charge of hardware support of SpaceWire-related upper protocols as SpaceWire-R. ${ }^{19)}$ So, if the SOI-SOC3 are used for the vertically assembled module in the future, it would be no need to have the FPGA outside the module. This would bring great merits to miniaturize the avionics.

The modules of Type A, B and C are connected by the socket on the evaluation board by turns. In this configuration, the following functions are tested.

\section{- Function of SpaceWire}

- Function of accessing the resisters inside the MPU

- Function of SRAM interface

- Function of SDRAM interface

All of the modules passed the above four tests successfully. These functional tests were executed in the vibration and thermal tests in order to confirm the healthiness of each module.

\subsection{Vibration tests}

Random vibration tests for the modules were carried out. The vibration was subjected in the three-axis directions: The $\mathrm{X}$ - and Y-directions are horizontal to the modules, and the Z-direction is vertical to the modules. Figure 7 (a) and (b) show the setup of the horizontal and vertical tests respectively. The vibration level was $15 \mathrm{Grms}$ in each direction considering the level of the ISAS small scientific satellites (e.g., ERG).

The tested samples were the modules of Type B and C. Both modules passed the functional tests described in 4.1 after the vibration tests successfully.

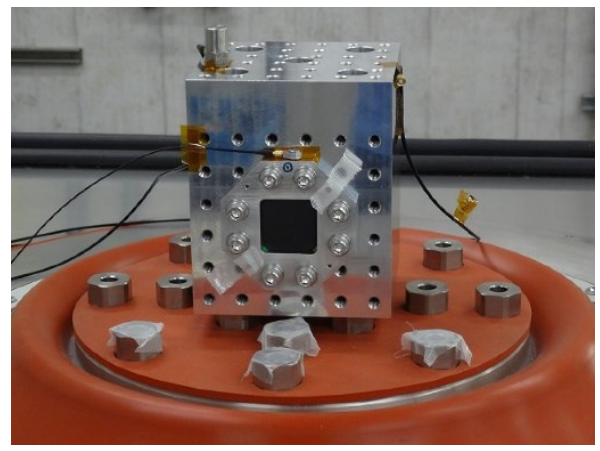

(a)

\subsection{Thermal tests}

The thermal tests of the modules were executed using a thermostat oven. As shown in Fig. 8, the modules together with the evaluation board were put into the thermostat oven. The tested samples were the modules of Type A and B.

In the first place, the settings of thermostat oven were the fixed temperatures: The high temperature was $125 \mathrm{degC}$ and the low temperature was $-55 \operatorname{deg}$. In both temperature cases, the modules passed the functional tests described in 4.1 successfully.

Then the thermal cycles were applied to the modules from 125 to $-55 \mathrm{degC}$. The number of cycle was ten. All during the cycles, the functional tests described in 4.1 were continued. The module of Type A passed all tests through the cycles. The module of Type B passed the SpaceWire, the resister accessing, and the SRAM interface tests through the cycles. In the SDRAM interface test of the Type B module, disconnection of one data bus line occurred between the MPU and the SDRAM. At the result of detailed inspections, the disconnection was found at the position of the wire bonding. Every connection by the flip chip bonding between the

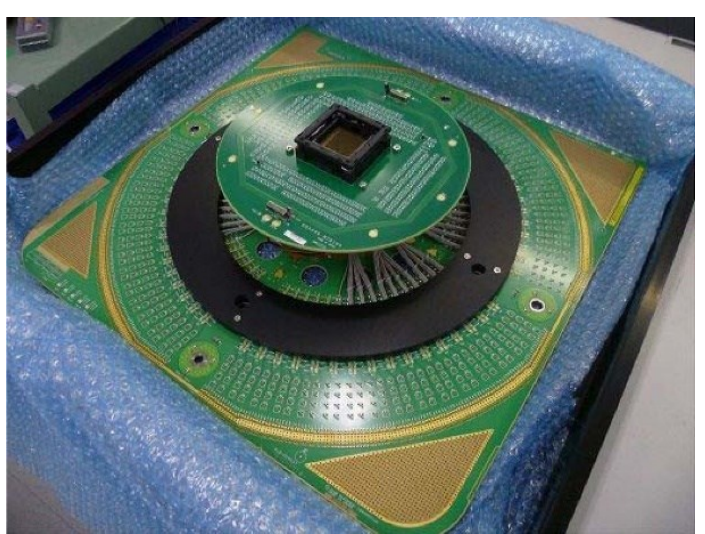

Fig. 6. Performance board to interface between the IC tester and the tested modules.

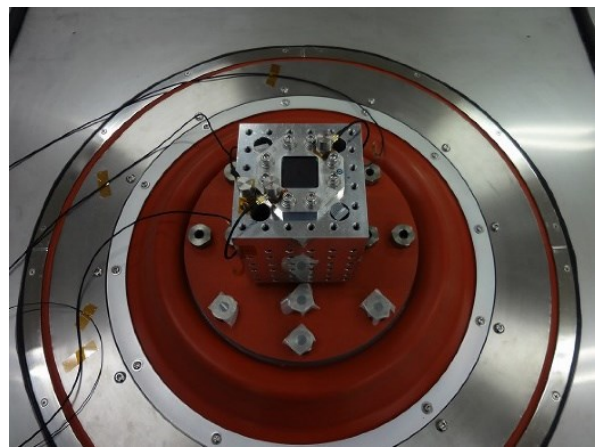

(b)

Fig. 7. Setup of the random vibration tests: (a) horizontal and (b) vertical directions respectively. 


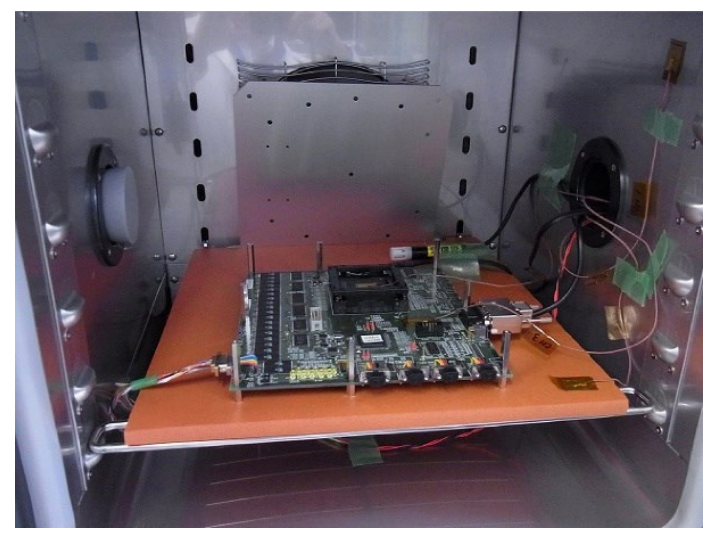

Fig. 8. Setup of the thermal tests: The vertically assembled module together with the evaluation board was put into the thermostat oven.

substrates and the bare chips, and by the room-temperature bonding between the layers of the substrates survives during and after the thermal cycles. As mentioned in the previous section, for the robustness of the wire bonding the thickness of the Au plating becomes the design parameter. The Au plating of the samples used in this test is as thick as about $1.3 \mu \mathrm{m}$. Some slipping of the wires would occur on such thin plating. Since the Silicon substrate is like a cantilever in the proposed modules, the ultrasonic load for the bonding is conducted due to the slipping of the wires so that the vibration of the substrate would deteriorate the bondability of the wires. Considering the diameter of the wire $(\sim 25 \mu \mathrm{m})$, doubling the thickness of the Au plating could make the bonding more robust. The validation of the effectiveness by increasing the thickness of the Au plating is left for future work.

\section{Conclusion}

In this paper, the novel scheme of the vertical assembly has been proposed in order to miniaturize the avionics onboard the spacecrafts. Since the Silicon substrates with the wiring patterns are stacked vertically by the room-temperature bonding, a variety of the existing heterogeneous bare chips can be used in the assembly. So in this scheme the advanced consumer technique is successfully applied, overcoming the restrictions of the space use such as the small volume production. The validation tests for the vertically assembled modules such as functional, vibration, and thermal tests, have been also carried out.

The proposed assembly scheme will be applied to the sensors which can be mounted on the Silicon substrates. So, using this technique, it can be expected to realize the one-chip camera with the CMOS image sensors or the one-chip IMU (Inertial Measurement Unit) with the MEMS gyros in the future.

\section{Acknowledgments}

This work was supported in part by a Grant-in-Aid for Strategic Development from the Committee for Space
Engineering in ISAS/JAXA

\section{References}

1) Matsuo, Y., Matsushima, H., Kuribayashi, M., Hihara, H., Kurumisawa, Tanaka, T., Watanabe, O., Fukuda, S., and Ishida, T.: Evaluation Result of the Consolidation Component, Proceeding of 60th Space Sciences and Technology Conference, Hakodate, Japan, JSASS-2016-4237, 2016 (in Japanese).

2) Saito, H., Hirahara, M., Mizuno, T., Fukuda, S., Fukushima, Y., Asamura, K., Nagamatsu, H., Tanaka, K., Sone, Y., Okuizumi, N., Mita, M., Uno, M., Yanagawa, Y., Takahara, T., Kaneda, R., Honma, T., Sakanoi, T., Miura, A., Ikenaga, T., Ogawa, K., and Masumoto, Y.: Small Satellite REIMEI for Auroral Observations, Acta Astronautica, 69 (2011), pp.499-513.

3) Fukuda, S., Mizuno, T., Sakai, S., Fukushima, Y., and Saito, H.: Integrated Satellite Control in REIMEI (INDEX) Satellite, Japan Soci. Aero. Space Sci., 57 (2009), pp. 25-31 (in Japanese).

4) Sawai, S., Fukuda, S., Sakai, S., Kushiki, K., Arakawa, T., Sato, E., Tomiki, A., Michigami, K., Kawano, T., Okazaki, S., Kukita, A., Miyazawa, Y., Ueda, S., Tobe, H., Maru, Y., Shimoji, H., Shimizu, Y., Shibasaki, Y., Shimada, S., Yokoi, T., Yabushita, T., Sato, K., Nakamura, K., Kuhara, T., Takami, T., Tanaka, N., and Furukawa, K.: Preliminary System Design of Small Lunar Landing Demonstrator SLIM, Aerospace Technology Japan, 17 (2018), pp. 35-43 (in Japanese).

5) Namikoshi, H., Sugawara, H., Chiba, A., Washino, R., Shuto, K., Shimizu, Y., Shibasaki, Y., Shimoji, H., Sato, K., Yokoi, T., Kono, J., Miuki, D., Aoki, Y., Sakai, S., Kushiki, K., Sawai, S., Fukuda, S., and Ishida, T.,: Preliminary System Design of SLIM, Proceeding of 62th Space Sciences and Technology Conference, Kurume, Japan, JSASS-2018-4081, 2018 (in Japanese).

6) Fukuda, S., Sawai, S., Sakai, S., Saito, H., Tohma, T., Takahashi, J., Toriumi, T., and Kitade, K.: Concept Study on a Flexible Standard Bus for Small Scientific Satellites, Space Technology Japan, 8 (2009), pp. 1-7 (in Japanese).

7) Nakaya, K., Fukuda, S., Sakai, S., Yamazaki, A., Uemizu, K., Toriumi, T., Takahashi, J., Maehara, M., Okahashi, T., and S. Sawai, Development of Flexible Standard Bus for ISAS/JAXA Small Scientific Satellite Series, Trans. JSASS Space Technology Japan, 10 (2012),pp.Tf_5-Tf_9.

8) Takahashi, T., Takashima, T., Fukuda, S., Kuboyama, S., Nomachi, M., Kasaba, Y., Tohma, T., Hihara, H., Moriyama, S., and Tamura, T.: Space Cube 2 - An Onboard Computer Based on Space Cube Architecture, International Spacewire Conference 2007, Dundee, Scotland, UK, pp.65-68, 2007.

9) European Cooperation for Space Standardization: SpaceWire Links, Nodes, Routers and Networks, ECSS-E-ST-50-12C, 2008.

10) European Cooperation for Space Standardization: SpaceWire Remote Memory Access Protocol, ECSS-E-ST-50-52C, 2010.

11) Hihara, H., Fukuda, S., Ishida, T., Tanaka, T., Watanabe, O., Matsuo, M., Kuribayashi, M., Matsushima, H., Shinozaki, K., and Yamada, T.: Innovative Miniaturization for Low Resource Interplanetary Exploration, International Spacewire Conference 2016, Yokohama, Japan, pp.89-93, 2016.

12) Takagi, H., Kikuchi, K., Maeda, R., Chung, T. R., and Suga, T.: Surface Activated Bonding of Silicon Wafers at Room Temperature, Appl. Phys. Lett., 68 (1996), pp.2222-2224.

13) Takagi, H., Maeda, R., Hosoda, N., and Suga, T.: Transmission Electron Microscope Observations of $\mathrm{Si} / \mathrm{Si}$ Interface Bonded at Room Temperature by Ar Beam Surface Activation, Jpn. J. Appl. Phys., 38 (1999), pp.1589-1594.

14) Hirose, K., Saito, H., Fukuda, S., Kuroda, Y., Ishii, S., Fukuoka, Y., and Takahashi, D.: Development of Radiation Hardening SOI-SRAM, Proceedings of the 3rd Space Science Symposium, Sagamihara, Japan, pp.285-289, 2003 (in Japanese).

15) Kuroda, Y., Shigeru, I., Takahashi, D., Kimura, S., Saito, H., and Hirose, K.: Development of Space and Commercial Dual-use Processor: Development of SOI-SOC, Japan Soci. Aero. Space 
Sci., 59 (2011), pp. 149-154 (in Japanese).

16) Takashima, T., Ogawa, E., Asamura, K., and Hikishima, M.: Design of a Mission Network System Using SpaceWire for Scientific Payloads Onboard the Arase Spacecraft, Earth, Planets and Space, 70 (2018), Article number: 71 (2018).

17) Narita, T., Taeda, M., Kato, M., Kusano, M., Masukawa, K., Ishida, T., Fukuda, S., Matsuzaki, K., Takahashi, T., Takada, M., Takada, H., and Nomachi, M.: High-Reliability Spacewire Engine
Implemented on SOISOC3 Microprocessor, International Spacewire Conference 2016, Yokohama, Japan, pp.75-78, 2016.

18) Ishida, T., Fukuda, S., Matsuzaki, K., Takahashi, T., Takada, M., Takada, H., Nomachi, M., Narita, T., Taeda, M., Masukawa, K., and Saso, K.: Software and SpaceWire Evaluation of SOI-SOC3, International Spacewire Conference 2016, Yokohama, Japan, pp.79-82, 2016.

19) Yamada, T.: SpaceWire-R, SCDHA 151-0.3, JAXA/ISAS, 2013. 